\title{
Thermal processing induced plastic deformation in GaAs wafers
}

\author{
P. Möck ${ }^{\mathrm{a}, *}$, Z.J. Laczik ${ }^{\mathrm{b}}$, G.R. Booker ${ }^{\mathrm{a}}$ \\ ${ }^{a}$ Department of Materials, University of Oxford, Parks Road, Oxford OX1 3PH, UK \\ ${ }^{\mathrm{b}}$ Department of Engineering Science, University of Oxford, Parks Road, Oxford OX1 3PJ, UK
}

\begin{abstract}
Different types of dislocation bundles were identified in the (001) GaAs substrates of III-V heterostructures. Comparisons of scanning infrared polariscopy images and X-ray transmission topograms showed a one to one correlation of stripes of reduced residual shear strain and dislocation bundles of the majority type. Visible-light interferometry and Makyoh topography, on the other hand, showed a slip-line distribution that is in correspondence to the distribution of dislocation bundles of the minority type(s). A new model for the plastic deformation of circular GaAs wafers during thermal processing is briefly outlined and its good agreement with the main experimental results demonstrated. C) 2001 Published by Elsevier Science B.V.
\end{abstract}

Keywords: X-ray topography; Infrared polariscopy; Plastic deformation; GaAs

\section{Introduction}

It is well known that thermal treatment induced plastic deformation in GaAs substrates is a key factor that reduces the yield of opto- and microelectronic devices in manufacturing processes on an industrial scale [1-3]. We observed severe plastic deformation in the GaAs substrates of epitaxial III-V compound semiconductor samples that were grown in molecular beam epitaxy (MBE) machines of three different makes [4]. Modifications to the sample holder of a user built MBE machine [5] resulted in the complete eradication of this unwanted plastic deformation. It should be noted that the observed plastic deformation was entirely caused by the thermal treatments used for the epitaxial growth, but bore no other relation to the epitaxial growth processes.

The aim of this paper is to give both a short overview of the main experimental results of our previous studies for which we used a variety of methods [4-7], and a brief outline of a new model that explains these observations reasonably well. A series of unused substrates, i.e. wafers as they were delivered from different vendors, was also assessed by the same methods $[4,7]$.

\footnotetext{
* Corresponding author. Present address: Department of Physics (MC 273), University of Illinois at Chicago, 845 W. Taylor Street, Chicago, IL 60607-7059, USA..

E-mail address: pmoeck@uic.edu (P. Möck).
}

These assessments proved that the observed plastic deformation effects were entirely due to the thermal treatments we employed in the MBE machines.

\section{Experimental details}

The substrates were mainly $0.45 \mathrm{~mm}$ thick, (001) oriented, undoped, two-inch diameter GaAs wafers that were grown by the vertical gradient freeze Bridgman technique. The thermal treatments that were used for the epitaxial growth typically involved temperatures of up to about $650^{\circ} \mathrm{C}$. Structural and thermal processing details of the samples and experimental details of our analyses are given elsewhere [4-7]. We used epitaxial III-V compound semiconductor samples for most of our study because they were readily available and, as stated above and demonstrated in [3], completely adequate for this purpose.

\section{Main experimental observations and discussion}

Fig. 1a-d show a scanning infrared polariscopy image, an X-ray transmission topogram, a Makyoh topogram, and a visible-light interferogram of the sample that has been analyzed in detail in [6]. Due to a corresponding spatial distribution between dislocation bundles with 'effective' Burgers vectors $\pm 1 / 2\langle 110\rangle$ 
(glide directions $\langle 110\rangle$ on inclined $\{111\}$ planes), and stripes of reduced residual shear strain, Fig. 1a and b, show predominantly a pseudo-symmetric, four-fold dislocation bundle set, that originated around the four $\langle 100\rangle$ peripheral areas and which we call the majority type set.

Conversely, Makyoh topography (Fig. 1c), visiblelight interferometry (Fig. 1d), and standard Nomarski microscopy [7] show a slip-line distribution that is in correspondence to the distribution of dislocation bundles of the minority type(s), which originated around $\langle 110\rangle$ peripheral areas.

A complete Burgers vector analysis of the majority type dislocation bundles showed that all dislocations in these bundles are of the $60^{\circ}$ type [6] and should therefore produce slip lines at the wafer surface that would be visible by the employed surface inspection techniques. There are, however, two different types of dislocations in each bundle of the majority type in about equal numbers. We suggest that the dislocations in the majority type bundles form in effect pairs, e.g. $\pm 1 / 2$ [101] and $\pm 1 / 2$ [01-1] 'pair up' to form $\pm 1 / 2$ [110], and the effective Burgers vector of these pairs does not possesses a component parallel to the surface normal.
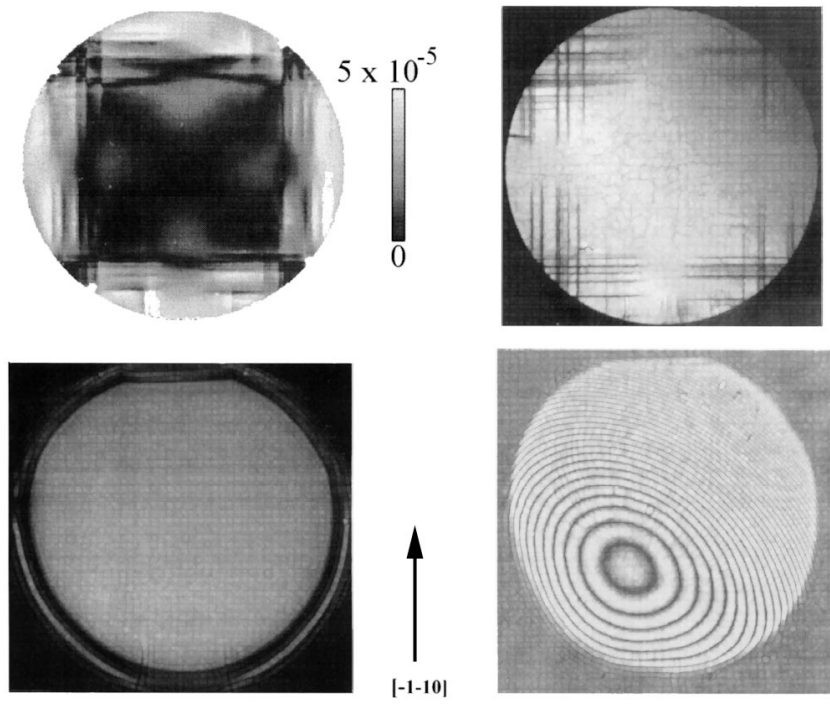

Fig. 1. Comparison of an (a) upper left, scanning infrared polariscopy image depicting residual shear strains, (b) upper right, (0-22) X-ray transmission topogram, (c) lower left, Makyoh topogram, (d) lower right, visible-light interferogram of the same sample. All images are in the same orientation and the major flats, i.e. [-1-10] directions, point up towards the top of the page. Majority type dislocation bundles with a four-fold pseudo-symmetry are clearly visible in the $\langle 100\rangle$ peripheral areas of (a) and (b). Minority type dislocation bundles are clearly visible at locations opposite to the major flats in (a)-(c). At the same places in (d), there are slight disturbances of the interference fringes, which correspond to step heights of about $30-50 \mathrm{~nm}$. Whole two-inch diameter wafers are depicted except for small mounting and imaging artifacts.
Dislocation bundles of the minority type(s), on the other hand, are assumed to be unpaired and should therefore possess as a consequence a Burgers vector component parallel to the wafer normal. This should result in slip lines that are visible by the employed surface inspection techniques.

The models given in $[1,2,9]$ describe the frequently occurring predominance of minority type dislocation bundles at the area of the major flat reasonably well, but are not applicable to majority type dislocation bundles since they predict a spatial distribution with an eightfold symmetry. Applying Curie's well-known symmetry principle [10,11], we found that the symmetry of this set is the point group $\overline{4} 2 \mathrm{~m}$ [6], which does not contain an eight-fold symmetry axis. The models mentioned above must therefore be oversimplifications.

\section{Model for thermal treatment induced plastic deformation in circular GaAs wafers}

Our new model is based on both the calculated resolved thermal shear stresses on the dislocation glide systems for a circular wafer and dislocations pairs as basic constituents of majority type dislocation bundles. The stress distribution during plastic flow is assumed to be independent of the number, density and spatial distribution of freshly introduced dislocations. The dependence of the stress distribution during plastic flow on grown-in dislocations is neglected. Hooke's law in its isotropic approximation is employed.

Assuming a tangentially uniform residual strain distribution, we calculated the critical resolved shear stresses on the eight actually operation slip systems, as given in [6], employing the formulae and material data given in [12]. These calculations predict for the outermost margin of the residual strain annulus (i.e. $4.75 \times$ $10^{-5}$, see Fig. 1a in [7]) two curves for the resolved thermal shear stresses $\tau_{1,2}$ and $\tau_{5,6}$ over an angular parameter that counts from the [100] direction (see Fig. 2 , where the subscripts are explained). Calculations for the other four operating slip systems lead to an analogous graph with an angular shift of $90^{\circ}$.

There are only four small angular regions where $\tau_{1,2}$ and $\tau_{5,6}$ possess values that are bigger than the experimentally derived threshold for dislocation generation [2] in the applicable deformation geometry. This explains that minority type dislocation bundles can glide in certain peripheral areas. Fig. 2 shows in good agreement to the experimental results [1-9] that the area where minority type dislocations can exist ranges from about $10^{\circ}$ to about $35^{\circ}$ around $\langle 110\rangle$ peripheral areas. Our model does not give an explanation for the frequently observed predominance of minority type dislocation bundles at the major flat, but this is to be expected from our assumption of a perfectly circular wafer. 


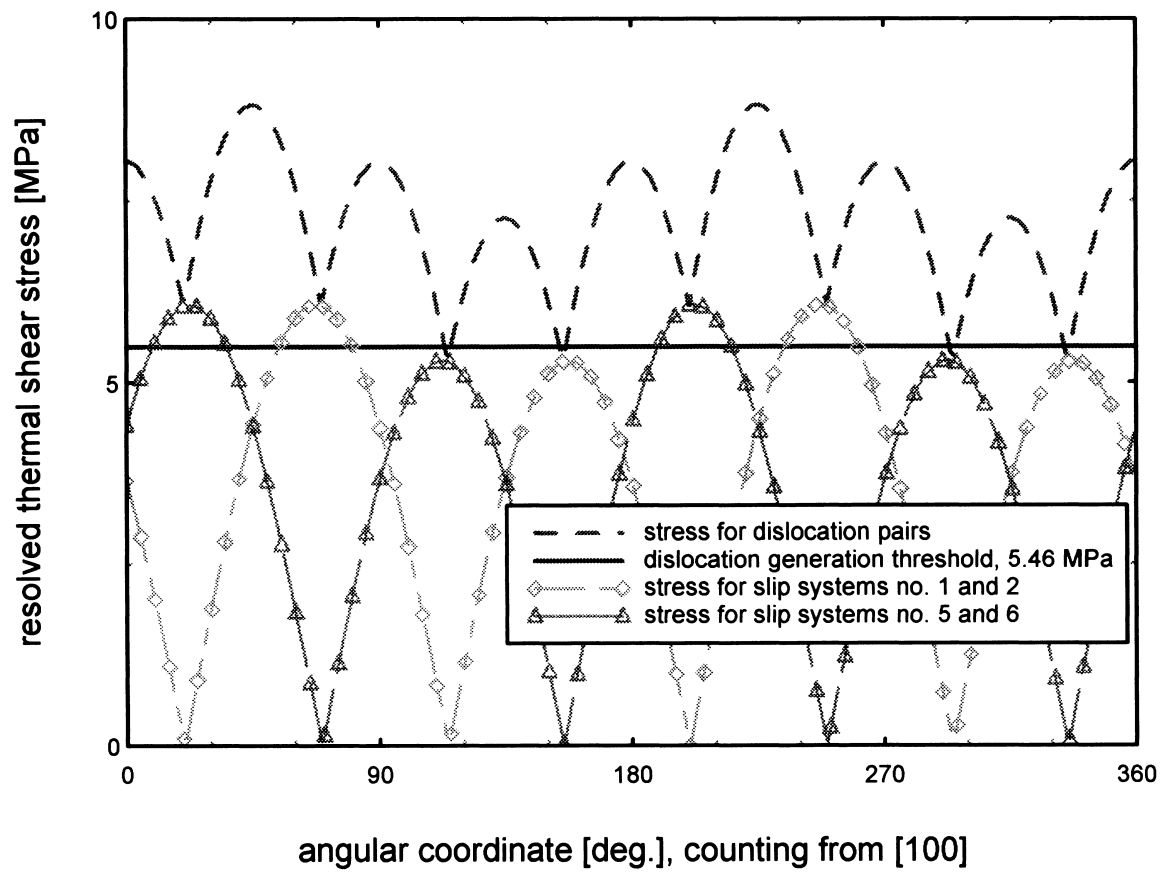

Fig. 2. Predicted resolved thermal shear stress as a function of an angular coordinate that counts from [100] for the slip systems No. 1: [0-11] (111), No. 2: [011] (-1-11), No. 5: [10-1] (111), and No. 6 [-10-1] (-1-11), and dislocation pairs (i.e. Nos. $1+5$ and $2+6)$.

The startling conclusion from Fig. 2 is, however, if dislocation 'pairing up' would not exist, a few minority type dislocation bundles would be all that is possible to relax the thermally induced strain. If we, however, take dislocation pairs into account, the situation changes completely.

The resolved thermal stresses $\tau_{1}+\tau_{5}=\tau_{2}+\tau_{6}$ for dislocation pairs are for nearly all angular regions above the threshold for dislocation glide. Thus, glide of dislocation pairs is possible nearly everywhere at the wafer edge. The locations where the glide of dislocation pairs will actually happen, however, are thought to be determined by the spatial distribution of the density of dislocation sources, which seem to be highest around the $\langle 100\rangle$ peripheral areas, Fig. 1a and b. Taking this distribution into account, the angular range of majority type dislocation bundles is, as Fig. 2 in good agreement to the experimental results [1-7,9] indicates, about $\pm 22^{\circ}$ around $\langle 100\rangle$ directions.

A two-fold symmetry follows directly from Fig. 2. Taking into account the other four actually operating slip systems, we obtain for undoped GaAs a four-fold pseudo-symmetry. The resolved thermal stress curves indicate that the further away a dislocation bundle of the majority type nucleates from an exact $\langle 100\rangle$ peripheral position, the shorter it will be.

For a residual strain of about $2.5 \times 10^{-5}$ (Fig. 1a in [7]) model calculations predict that glide of majority type dislocation bundles from $\langle 100\rangle$ peripheral areas ceases since the resolved thermal shear stresses on dislocation pairs fall below the critical thermal stress for dislocation generation. This is as well in good agreement to the experimental observations [7].

Contrary to the predictions of [9], the four slip systems that involve screw dislocations are, as the experiments [6] and our calculations show, not activated. This is because their resolved thermal shear stresses are for all experimentally observed residuals strain levels (Fig. 1 in [7]) well below the critical thermal stress for dislocation generation. A comprehensive description of our model and a more detailed comparison between experiment and theory will be given elsewhere.

\section{Conclusions}

Different types of dislocation bundles have been identified by means of a combination of scanning infrared polariscopy, X-ray transmission topography, visible-light interferometry and Makyoh topography. In order to explain the experimental observations, we propose that the dislocations in the majority type dislocation bundles pair up, cancelling surface steps and slip lines to some extent. A new model for thermal treatment induced plastic deformation of circular GaAs wafers has briefly been outlined. The predictions of this model are in good agreement to the main experimental observations. 


\section{Acknowledgements}

We wish to thank M. Yamada and M. Fukuzawa, Kyoto Institute of Technology, who kindly provided the scanning infrared polariscopy image. Financial support from the British Engineering and Physical Science Research Council is gratefully acknowledged.

\section{References}

[1] S. Sawada, H. Yoshida, M. Kiyama, H. Mukai, R. Nakai, T. Takebe, M. Tatsumi, M. Kaji, K. Fujita, Technical Digest-IEEE GaAs Integrated Circuit Symp. 74 (1996) 50.
[2] M. Kiyama, T. Takebe, K. Fujita, Inst. Phys. Conf. Ser. 155 (1997) 945.

[3] M. Yamada, M. Fukuzawa, T. Kawase, M. Tatsumi, K. Fujita, Inst. Phys. Conf. Ser. 145 (1996) 447.

[4] P. Möck, J. Cryst. Growth (accepted for publication).

[5] P. Möck, G.W. Smith, Cryst. Res. Technol. 35 (2001) 541.

[6] P. Möck, J. Appl. Cryst. 34 (2001) 65.

[7] P. Möck, M. Fukuzawa, Z. Laczik, G.W. Smith, G.R. Booker, M. Yamada, M. Herms, B.K. Tanner, Inst. Phys. Conf. Ser. 164 (1999) 67.

[8] M. Yamada, Rev. Sci. Instrum. 64 (1993) 1815.

[9] M. Yamada, M. Fukuzawa, K. Ito, Inst. Phys. Conf. Ser. 155 (1997) 901.

[10] P. Curie, J. Physique 3 (1894) 393.

[11] L.A. Shuvalov, Modern Crystallography IV, Physical Properties of Crystals, Springer Series in Solid-State Science, vol. 37, Springer, Berlin, 1988, pp. 46-49.

[12] P. Möck, Cryst. Res. Technol., 35 (2000) 529. 